\title{
A negative correlation between the induction of long-term potentiation and activation of immediate early genes
}

\author{
Steven S. Schreiber ${ }^{1}$, Stephen Maren ${ }^{2}$, Georges Tocco ${ }^{2}$, Tracey Jo Shors ${ }^{2}$ and \\ Richard F. Thompson ${ }^{2}$ \\ 'University of Southern California, School of Medicine, Department of Neurology, Los Angeles, CA 90033 (U.S.A.) and ${ }^{2}$ Neurosciences \\ Program, University of Southern California, Hedco Neurosciences Building, Los Angeles, CA 90089-2520 (U.S.A.)
}

(Accepted 9 April 1991)

Key words: Long-term potentiation; Immediate early gene; c-fos; zif/268; Dentate gyrus

\begin{abstract}
In the present study we examined the relationship between the induction of long-term potentiation (LTP) in the dentate gyrus of anesthetized rats and activation of immediate early genes (IEGs; c-fos and zifl268) using several different high-frequency stimulation paradigms. Stimulation parameters that effectively induced LTP were not associated with IEG activation. Conversely, stimulation parameters that failed to induce LTP consistently resulted in IEG activation. These results suggest that there is a negative correlation between IEG activation and LTP, and that activation of IEGs is neither necessary nor sufficient for the induction of LTP.
\end{abstract}

Immediate early genes (IEGs) are characterized by their rapid and transient induction in response to a wide variety of extracellular stimuli both in vitro and in vivo $^{7,10}$. There is increasing evidence that the proteins encoded by these genes act as transcription factors, regulating long-term changes within the central nervous system ${ }^{7,10}$. Indeed, IEG activation has been postulated to play a role in long-term potentiation (LTP), a long-lasting enhancement of synaptic efficacy occurring at excitatory synapses in the mammalian brain following brief rrains of high-frequency electrical stimulation appiied to afferent pathways ${ }^{\mathbf{1 , 6}}$. Consistent with this hypothesis, it has been reported that high-frequency stimulation of the perforant path results in the activation of several IEGs in the rat dentate gyrus ${ }^{2,12}$. The most consistently induced IEG, zif/268 (also known as NGFI-A), correlated with the induction of LTP. Because both LTP and IEG induction require the activation of the $N$-methyl-D-aspartate (NMDA) receptor ${ }^{3,8,11}$, it was suggested that IEG activation is necessary for LTP induction and expression ${ }^{2,12}$. However, data from earlier reports suggests that IEG activation is correlated not with LTP, but with highfrequency electrical stimulation ${ }^{4,5}$. Therefore, in the present study we examined the relationship between high-frequency stimulation, LTP induction, and IEG activation at rat perforant path-granule cell synapses in vivo.
Adult Long-Evans rats (250-300 g) anesthetized with sodium pentobarbital $(60 \mathrm{mg} / \mathrm{kg})$ were implanted stereotaxically with a recording electrode in the hilus of the dentate gyrus $(3.3 \mathrm{~mm}$ posterior, $2.4 \mathrm{~mm}$ lateral, and 2.8-3.0 mm ventral to bregma) and a bipolar stimulating electrode in the ipsilateral medial perforant path $(8.1 \mathrm{~mm}$ posterior, $4.4 \mathrm{~mm}$ lateral, and $2.5-4.0 \mathrm{~mm}$ ventral to bregma). The electrodes consisted of Epoxylite-coated stainless-steel pins with recording (50 $\mu \mathrm{m})$ and stimulating surfaces $(500 \mu \mathrm{m})$ formed by remioving the insulation at the tips. Electrophysiological testing (100 $\mu$ s putses at $0.05 \mathrm{~Hz}$; voltage adjusted to elicit an approximately $1 \mathrm{mV}$ population spike [PS]) began after stable dentate hilar field potentials evoked by perforant path stimulation had been maintained for at least $\mathbf{3 0} \mathrm{min}$. Evoked responses were collected $20 \mathrm{~min}$ prior to and $30 \mathrm{~min}$ following high(tetanic) or low-frequency stimulation. Low intensity high-frequency stimulation consisted of either two $1 \mathrm{~s} 100$ Hz trains separated by $\mathbf{3 0}$ s (HF; $n=4)$ or ten $\mathbf{4 0} \mathrm{ms} \mathbf{4 0 0}$ $\mathrm{Hz}$ bursts at $5 \mathrm{~Hz}$ (theta; $n=6$ ) delivered at a voltage sufficient to elicit a $1 \mathrm{mV}$ PS. High-intensity highfrequency stimulation (theta*; $n=6$ ) consisted of theta stimulation delivered at a voltage twice that which elicited a maximal PS. Low-frequency stimulation (LF; $n$ $=5$ consisted of $1 \mathrm{~Hz}$ stimulation for $200 \mathrm{~s}$. The evoked responses recorded during pre- and post-tetanus periods were averaged; subjects exhibiting a $10 \%$ or greater

Correspondence: S.S. Schreiber, Department of Neurology, University of Southern California School of Medicine, $2025 \mathrm{Zonal}$ Avenue, MCH 142, Los Angeles, CA 90033, U.S.A. 


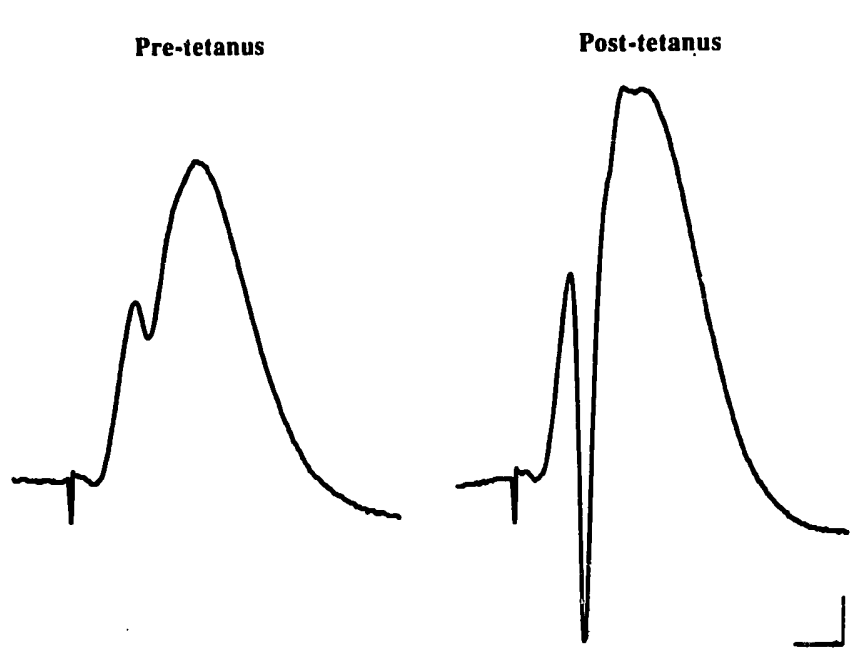

Fig. 1. Representative pre- and post-tetanus averaged field potentials from a subject exhibiting robust long-term potentiation. Each trace is an average of 5 evoked potentials recorded from the hilar region of the dentate gyrus. Calibrations: $2.78 \mathrm{~ms}, 1.44 \mathrm{mV}$.

post-tetanus increase in the excitatory postsynaptic potential (EPSP) slope and a $60 \%$ or greater increase in PS amplitude were scored as LTP positive. Representative pre- and post-tetanus averaged evoked responses for a subject exhibiting robust LTP appear in Fig. 1.

One hour following stimulation the rats were decapitated, their brains rapidly removed, frozen in $-20^{\circ} \mathrm{C}$ isopentane, and stored at $-70^{\circ} \mathrm{C}$ until sectioning. Coronal sections $\left(10 \mu \mathrm{m}\right.$ thick) were cut at $-15^{\circ} \mathrm{C}$ in a cryostat. The sections were thaw-mounted on chromealum/gelatin coated slides and stored at $-70^{\circ} \mathrm{C}$.

In situ hybridization, including the preparation of zif/268 and c-fos riboprobes, was performed as previously described ${ }^{9}$. Briefly, brain sections were fixed in $4 \%$ paraformaldehyde. Prehybridization treatment included a brief wash in $0.1 \mathrm{M}$ triethanolamine and acetic anhydride. The sections were hybridized to $\left[{ }^{35}\right.$ S]UTP

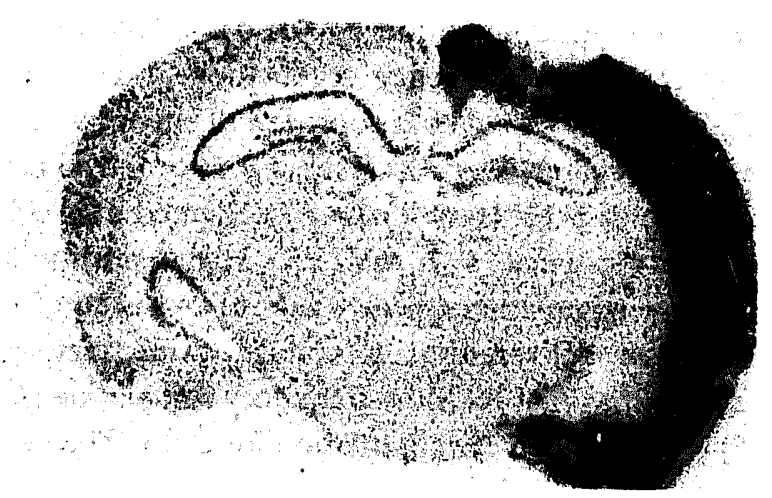

Fig. 2. Hippocampal IEG induction is absent in an animal that exilibited LTP. In situ hybridization using zif/268 probe on a representative section from an animal that received low intensity patterned stimulation (theta). Note neocortical induction ipsilateral to the site of stimulation.

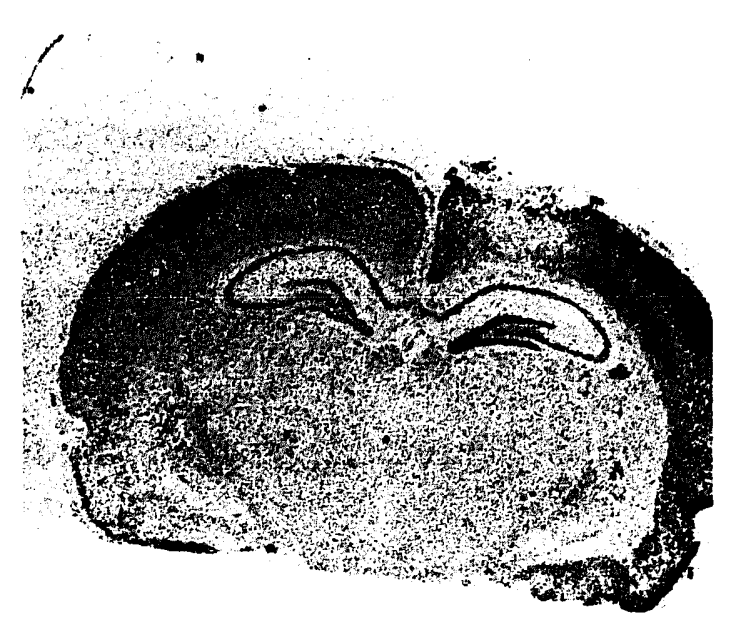

Fig. 3. Hippocampal IEG induction is present in an animal that did not exhibit LTP. In situ hybridization using zif/268 probe on a representative section from an animal that received high-intensity patterned stimulation (theta*). Note increased signal over the dentate gyrus and neocortex ipsilateral to the site of stimulation.

labeled riboprobes for $3 \mathrm{~h}$ at $50^{\circ} \mathrm{C}$. The final highstringency wash was performed in $0.1 \times \mathrm{SSC}$ at $60^{\circ} \mathrm{C}(1$ $\times \mathrm{SSC}=0.15 \mathrm{M}$ sodium chloride, $0.015 \mathrm{M}$ sodium citrate). After dehydration, dried sections were exposed to Kodak X-omat film for 3-7 days. Hybridization of tissue sections after pretreatment with RNAase $A$ or with sense probes resulted in non-specific background labeling (data not shown). Gene induction was quantified by comparing optical density (O.D.) measurements (BRAIN, Drexel University) between the ipsilateral (stimulated) and contralateral (unstimulated) dentate gyri; measurements were made on duplicate sections. An OD increase of greater than $15 \%$ on the ipsilateral side was scored as a positive induction.

The results are depicted in Table I. Of the shamoperated animals (sham; electrode implantation without electrical stimulation), IEG induction was observed in 2 out of 6 animals (1 rat exhibited zif/268 induction without c-fos induction). In animals receiving low-frequency stimulation (LF) neither LTP nor gene induction was observed. Relatively low intensity high-frequency stimulation (HF) induced LTP in 2 out of 4 animals; none of the animals, however, exhibited IEG induction. Low-

\section{TABLE I}

Relationship between stimulation parameters, LTP, and IEG MRNA

\begin{tabular}{lllll}
\hline Group & $n$ & LTP & zifl268 & $c$-fos \\
\hline Sham & 6 & - & 2 & 1 \\
LF & 5 & 0 & 0 & 0 \\
HF & 4 & 2 & 0 & 0 \\
Theta & 6 & 6 & 1 & 1 \\
Theta* & 6 & 0 & 5 & 4 \\
\hline
\end{tabular}


intensity patterned stimulation (theta) consistently induced LTP (6 out of 6 animals); IEG activation occurred in only 1 animal. Fig. 2 shows a coronal section from a rat (theta group) that exhibited LTP in the absence of IEG induction. When the patterned stimulation was delivered at a high intensity (theta*), LTP induction did not occur. IEG activation, however, was observed in 5 of the 6 animals (1 rat exhibited zif/268 induction without c-fos induction). Fig. 3. shows a coronal section from a subject (theta* group) that did not exhibit LTP, but exhibited prominent IEG activation. IEG induction in the dentate gyrus (when it occurred) was always ipsilateral to the stimulating electrode; unilateral cortical IEG induction occurred in all of the subjects.

The results of the present study indicate that there is a negative correlation bettveen IEG activation and the induction of LTP in the dentate gyrus of anesthetized rats. That is, stimulation parameters that induced LTP were not associated with zif/268 or c-fos activation, whereas, high intensity patterned stimulation that failed to induce LTP was associated with IEG activation. These results are in agreement with studies that have reported a poor correlation between c-fos activation and LTP induction ${ }^{2,4,5,12}$. However, the dissociation between ziff 268 activation and LTP observed in the present study is not in accordance with data from recent studies that have examined zif/268 activation following high-frequency stimulation. Both Cole et al. ${ }^{2}$ and Wisden et al. ${ }^{12}$ have

1 Bliss, T.V.P. and Lomo, T., Long-lasting potentiation of synaptic transmission in the dentate area of the anesthetized rabbit following stimulation of the perforant path, J. Physiol., 232 (1973) 331-356.

2 Cole, A.J., Saffen, D.W., Baraban, J.M. and Worley, P.F., Rapid increase of an immediate early gene messenger RNA in hippocampal neurons by synaptic NMDA receptor activation, Nature, 340 (1989) 474-476.

3 Collingridge, G.L., Kehl, S.J. and McLennan, H., Excitatory amino acids in synaptic transmission in the Schaffer-commissural pathway of the rat hippocampus, J. Physiol., 334 (1983) 33-46.

4 Douglas, R.M., Dragunow, M. and Robertson, H.A., Highfrequency discharge of dentate granule cells, but not long-term potentiation, induces c-fos protein, Brain, Res., 464 (1988) 259-262.

5 Dragunow, M., Abraham, W.C., Goulding, M., Mason, S.E., Robertson, H.A. and Faull, R.L., Long-term potentiation and the induction of c-fos mRNA and proteins in the dentate gyrus of unanesthetized rats, Neurosci. Lett., 101 (1989) 274-280.

6 Goelet, P., Castellucci, V.F., Schasher. S. and Kandel, E.R., The long and short of long-term memory - a molecular framework, Nature, 322 (1986) 419-422.

7 Morgan, J.I. and Curran, T., Role of the immediate-early genes, reported a high correlation between ITP induction and zif/268 activation in the dentate gyrus of anesthetized rats. One reason for the discrepancy may be the variability across laboratories in the stimulation protocols that are used for LTP induction. For example, the patterned stimulation used in the present study was applied over a relatively brief interval ( $2 \mathrm{~s})$, whereas other groups have used longer periods (1-3 $\mathrm{min}$ ) and/or different intraburst interval frequencies to deliver an equivalent number of pulses. Nevertheless, this does not reconcile the fact that in the present study IEG induction was most consistently observed following an inordinately high stimulation intensity, an intensity that was ineffective in inducing LTP. In the study conducted by Cole et al. $^{2}$, the most effective stimulation intensity for inducing both LTP and zif/268 was one at which a maximal PS was elicited. The results of the present study reveal that at intensities much higher than this (theta*), the induction of IEGs occurs in the absence of LTP, whereas at lower intensities (theta), LTP induction occurs in the absence of IEG activation, Thus, it may be the case that different patterns of intensities of stimulation induce quaiitatively different forms of LTP, some of which are associated with IEG induction.

This study was supported by grants from the National Institutes of Health (NS01337 to S.S.S., AG05142 and AG05500 to R.F.T.) and the McKnight Foundation (R.F.T.).

c-fos and c-jun, in stimulus transcription coupling in the mammalian central nervous system. In L.T. Landmesser (Ed.), The Assembly of the Nervous System, Alan R. Liss, New York, 1989, pp. 235-246.

8 Morris, R.G.M., Anderson, E., Lynch, G.S. and Baudry, M., Selective impairment of learning and blockade of long-term potentiation by an $\boldsymbol{N}$-methyl-D-aspartate receptor antagonist, AP5, Nature, (1986) 774-776.

9 Schreiber, S.S., Tocco, G., Shors, T.J. and Thompson, R.F., Activation of immediate early genes after acute stress, iveuroReport, 2 (1991) 17-20.

10 Sheng, M. and Greenberg, M.E., The regulation and function of c-fos and other immediate early genes in the nervous system, Neuron, 4 (1990) 477-485.

11 Szekely, A.M., Barbaccia. M.L., Alho, H. and Costa, E., In primary cultures of cerebellar granule cells the activation of $N$-methyl-D-aspartate-sensitive glutamate receptors induces c-fos mRNA expression, Mol. Pharmacol., 35 (1989) 401-408.

12 Wisden, W., Errington, M.L., Williams, S., Dunnett, S.B., Waters, C., Hitchcock, D., Evan, G., Bliss, T.V.P. and Hunt, S.P., Differential expression of immediate early genes in the hippocampus and spinal cord, Neuron, 4 (1990) 603-614. 